\section{Values and Psychiatric Diagnosis}

By John Z. Sadler. Oxford: Oxford University Press. 2004.540 pp. $€ 34.95$ (pb).

ISBN 0198526377

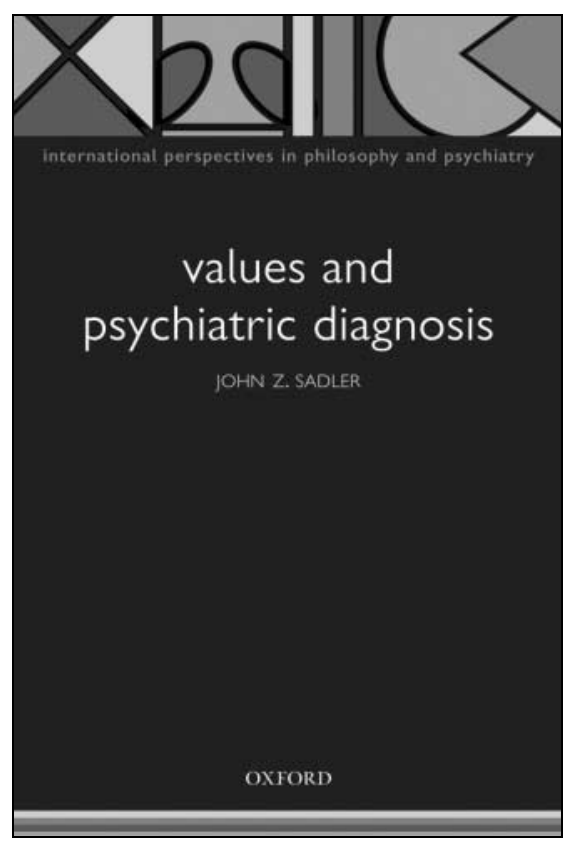

For more than ten years Sadler has been thinking about the ways in which psychiatric classification is imbued with judgements of value, even when it purports to be objective. Operational definitions of psychiatric disorders are intended to be universal, in some sense scientific, and as such should do away with value judgements. Sadler, whose massive scholarship is demonstrated in this book, shows the extent to which such judgements persist and why this is inevitable.

'Massive' is not hyperbole. The message that psychiatric diagnosis involves values is not too difficult to convey. What Sadler shows, however, is how values are involved, not just in the wording of particular diagnostic categories, but at every step of the way. They are involved in the very idea of pinning down psychiatric illnesses and placing them in categories: the determination that this can be done in a scientific manner involves 'value-commitments'. How the enterprise of creating a classificatory system is undertaken (e.g. with openness to non-psychiatric participation) is itself a political matter involving ethical and pragmatic values. Sadler lays bare the ways in which our world views - involving culture, religion, sex and gender - can shape our definitions of mental disorder.
This was most starkly shown in 1973 when, in America, homosexuality was voted not to be a mental disorder. How this is squared with the underpinning essentialism of nosology - according to which diseases have an invariant nature - shows the complexity of things; because an essence should be found, not voted in or out (which sounds more like the social construction of disorder). What emerges is the importance of our ontological assumptions: where we stand on how things are in the world.

It has to be said that this is a long book. Nevertheless, its individual chapters could be highly recommended to specific groups for various purposes - none more so than the chapter on technology, in which the need for a balance between technological practice (which is efficient, productive and economical) and poietic practice (to do with creativity, tradition, nature, connectedness) is suggested. Sadler, who is one of the main movers in the field of the philosophy of psychiatry, emphasises the need for balance between scientific and philosophical understandings, which is crucial if clinical practice is to aspire to excellence.

Julian C. Hughes Consultant in Old Age Psychiatry and Honorary Clinical Senior Lecturer, NorthTyneside General Hospital and the Institute for Ageing and Health, University of Newcastle, North Tyneside General Hospital, Rake Lane, North Shields NE29 8NH, UK.

E-mail: j.c.hughes@ncl.ac.uk

\section{Manual of Psychiatric Care for the Medically III}

Edited by Antoinette Ambrosino Wyszynski \& Bernard Wyszynski. Washington, DC:

American Psychiatric Publishing. 2005. 416 pp. US $\$ 64.00$ (pb). ISBN I585621।88

This is a good book. Don't be deterred by the flimsy cover and coil binding, which I viewed with great sceptism on its arrival. It seemed an unlikely candidate to survive the rigours of life chez Garden. However, soon the advantages of the design become apparent; it is very compact and pages with useful scales and instruments could be reproduced with ease (subject to copyright). You can find your way around. The logical structure, with largely predictable and systematic coverage of different systems, is an advantage both for psychiatrists with limited medical knowledge and for physicians with little mental health experience.

It is indeed a manual. In each section there is a useful clinical summary of conditions described. It combines basic background medical information, summaries of psychiatric aspects of conditions with practical 'how do I . . . ?' questions about psychotropic medication, and relevant scales, their uses and limitations in each clinical setting. Inclusion of a section on obstetric patients, for whom rapid, relevant and safe response may be crucial, is particularly useful for psychiatrists without the luxury of a perinatal mental health service. The section on capacity is a timely bonus for UK practitioners, since objective assessment of capacity will become highly topical when the Incapacity Act comes into force.

However, the book does have limitations for the UK reader. There is information about drugs that are unavailable in the UK, and the use of many abbreviations unfamiliar to the non-US practitioner requires the reader to make frequent reference to the key. Furthermore, several important subjects are not covered. For example, medically unexplained symptoms, particularly topical in British liaison psychiatry, are addressed by system rather than as a collective problem, and relevant psychotherapeutic interventions, such as reattribution, receive little if any mention. In addition, the emphasis is on medical rather than psychotherapeutic intervention, perhaps reflecting differences in practice on either side of the Atlantic. It is notable that the chapter addressing psychological issues in medical patients is only three pages long.

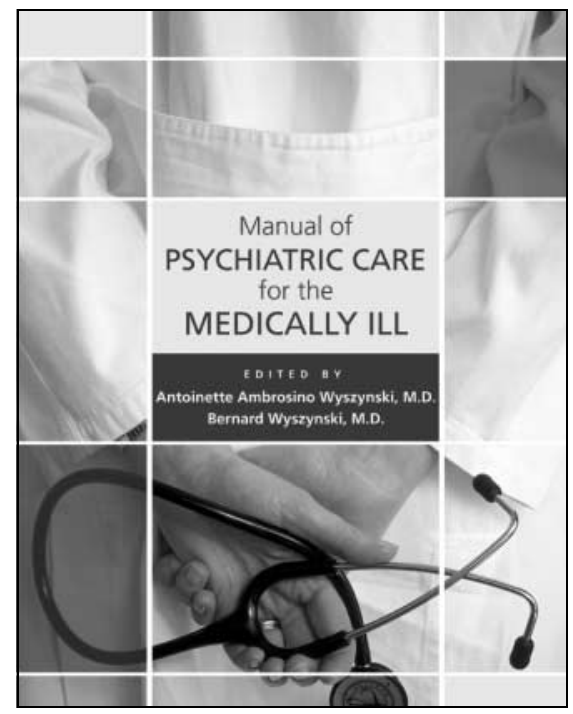

